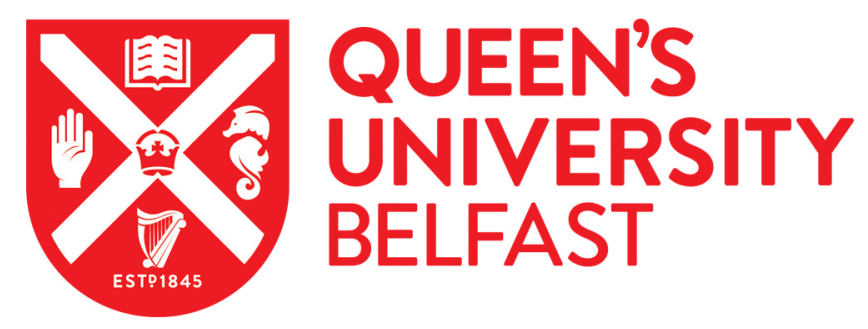

\title{
Physical Layer Biometrics using Antennas for Secure Wearable Wireless Communication
}

Conway, G. A., McAllister, J., Saadat, W., \& Raurale, S. (2019). Physical Layer Biometrics using Antennas for Secure Wearable Wireless Communication. IEEE Transactions on Antennas and Propagation, 67 (4), 2677 2686. https://doi.org/10.1109/TAP.2018.2889163

Published in:

IEEE Transactions on Antennas and Propagation

Document Version:

Peer reviewed version

Queen's University Belfast - Research Portal:

Link to publication record in Queen's University Belfast Research Portal

Publisher rights

Copyright 2018 IEEE. This work is made available online in accordance with the publisher's policies. Please refer to any applicable terms of use of the publisher.

\section{General rights}

Copyright for the publications made accessible via the Queen's University Belfast Research Portal is retained by the author(s) and / or other copyright owners and it is a condition of accessing these publications that users recognise and abide by the legal requirements associated with these rights.

Take down policy

The Research Portal is Queen's institutional repository that provides access to Queen's research output. Every effort has been made to ensure that content in the Research Portal does not infringe any person's rights, or applicable UK laws. If you discover content in the Research Portal that you believe breaches copyright or violates any law, please contact openaccess@qub.ac.uk. 


\title{
Physical Layer Biometrics using Antennas for Secure Wearable Wireless Communication
}

\author{
Waqar Saadat, Sumit A. Raurale, Student Member, IEEE, Gareth A. Conway, Member, IEEE, John. McAllister, \\ Senior Member, IEEE
}

\begin{abstract}
This paper combines a study of human body morphology with physical layer characteristics to introduce a novel biometric identity feature for security in wearable communication applications. The physical layer characteristics of close fitting wearable devices are shown to vary in a unique manner through electromagnetic interactions between the tissue morphology and the antenna. Experimental measurement results demonstrate the new biometric concept using return loss characteristics to identify individuals on multiple body parts. An optimised directional coupler design is implemented with the antenna to optimize the characteristic feature detection range for human identification. Experiments conducted on human subjects using a prototype standalone test-bed and sensing circuitry at $2.45 \mathrm{GHz}$, shows that, classification accuracies of over $98 \%$ are achieved for stationary subjects and $93 \%$ for mobile subjects. The new physical layer biometric, has the potential to be used for authentication and authorization by using return loss as an indicator for secure user applications, using circuitry already implemented in wireless wearable communication systems.
\end{abstract}

Index Terms-biometric, antenna characteristics, return loss, directional coupler, wearable antenna, physical layer security.

\section{INTRODUCTION}

Biometrics and key generation have gained great attention in wireless communication in the past decade to improve system and data security [1], [2]. Biometric sensing is based on authentication using human body morphology or physiology and is adopted in different systems as a form of human identification and access control [3], [4]. Biometric-based physical layer security has been driven by secure mobile banking and cloud-based social media applications [5]. Biometrics have shown great potential, and are successfully being used in advanced security systems to provide unique protection from hacking [1]. Now, research is focused on investigating new biometric add-ons, which can be captured in real time, anywhere and everywhere [3]. Being able to integrate this capability with everyday life, often means that the devices need either, to be placed everywhere in convenient, strategic locations, or alternatively, that the user carries or wears their

Manuscript received 13 June 2018.

This work was supported by the Department for the Economy (DfE) NI, UK.

Waqar Saadat and Gareth A. Conway are with the Centre for Wireless Innovation (CWI), Institute of Electronics, Communications and Information Technology (ECIT), The Queens University of Belfast, Queens Road, Belfast, BT3 9DT, UK. (e-mail: wsaasat01@qub.ac.uk; g.conway@qub.ac.uk)

Sumit A. Raurale and John McAllister are with the Centre for Data Science and Scalable Computing (DSSC), Institute of Electronics, Communications and Information Technology (ECIT), The Queens University of Belfast, Queens Road, Belfast, BT3 9DT, UK. (e-mail: sraurale01@qub.ac.uk; jp.mcallister@qub.ac.uk). own (wearable) identification hardware as in Fig. 1, which is the theme of this work. The concept is that, from the dynamic antenna and nearfield interaction features, the user Alice or Bob could be identified and authenticated (Fig. 1) with only physical layer characteristics. The mobile and dynamic nature of the human body, consequently forces challenging physical requirements on these types of wearable systems. These include minimal weight, flexibility, conformability, and minimal volume requirements [6]. Hence, there is a reluctance to add additional circuitry for security, to an already relatively bulky system. Biometrics attained from physical layer characteristics offer significant usability advantages such as reducing or removing the need for additional hardware, as the physical layer hardware is already in place for wireless communication. Other biometric methods such as fingerprint scanning, retina scanners and DNA detectors, not only require additional hardware in remote wearable wireless systems, but also require the user to perform an action, which differentiates them from physical layer biometric approach, which requires no user interaction. Furthermore, to meet application demands, systems are now expected to communicate wirelessly so that the device operates unambiguously, creating another dimension of complexity and uncertainty.

An attractive, emerging application in 5G is direct Bodyto-Body (B2B) communications. Authenticating the target user is critical in obtaining a more secure link. Biometric enabled security has the potential to meet these performance requirements, particularly for close-fitting wearable systems. Security implementation on the physical layer is an open research question for the next generation, 5G, with aims to reduce computational cost and size of the system [7], yet provide enhanced performance.

Human radio biometric information has been analysed and demonstrated through propagating WIFI signals by capturing channel state information and separating them using time reversal techniques [4]. The presence of a user is identified by detecting patterns in channel characteristics, but identification features are sensitive to environmental changes. A mm-wave sensor was developed to identify humans movement through body-centric channel characteristics, which consisted of two transmit antennas and four receive antennas [8]. The received signal strength (RSSI) was used to recognize user gait and authenticate each individual at the same time through three radio channel features, the time series, auto-correlation function and level crossing rate [9]. The channel characteristic $\left|S_{21}\right|$ was used to identify humans through their palms by optimising two horn antennas at $28 \mathrm{GHz}$ [10]. The scattering 


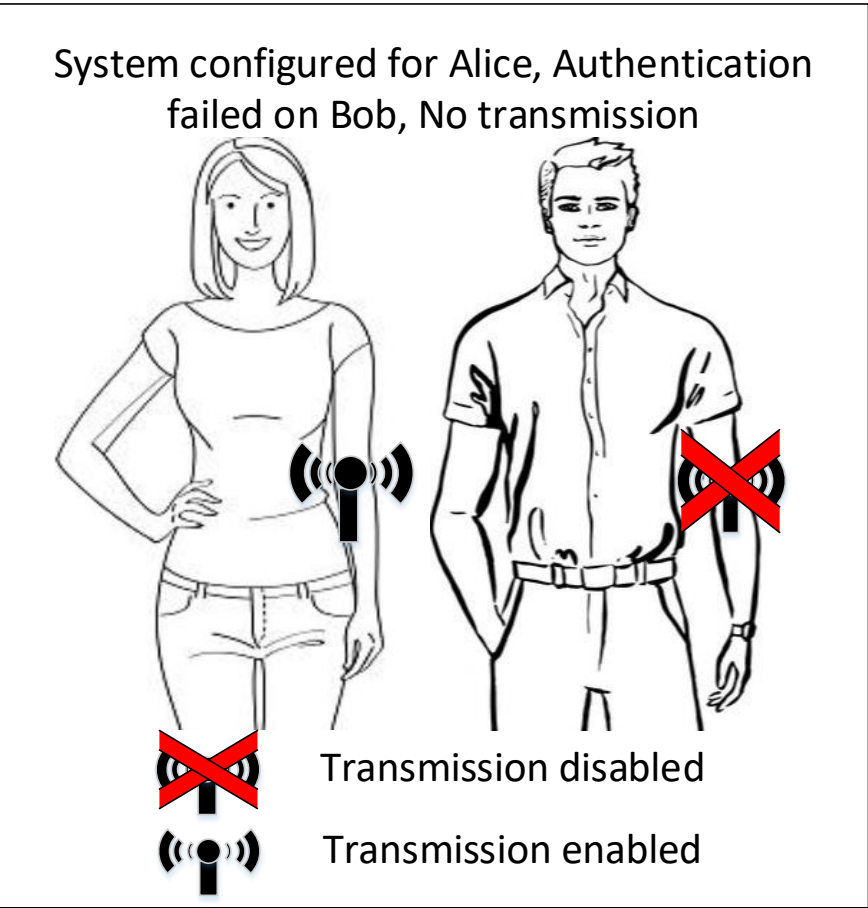

Fig. 1. Physical layer security wearable antenna implementation concept.

parameters were used as biometric, identifying human motion activities using line-of-sight and on-body creeping waves. The scattering parameters were also used to identify a dementia disorder by optimizing human movement sensing techniques [11]. The research shows that it was possible to define different human body positions by analysing the magnitude and phase of a creeping wave at different frequencies. However, the work did not look at identifying the user from other users. Interestingly [12], concluded that, measurement at $915 \mathrm{MHz}$ provided higher accuracy for body movement activities as compared with $433 \mathrm{MHz}$ and $2.4 \mathrm{GHz}$ [12]. In an alternative study presented in [13], four types of patch antennas were implemented on a transmitter and receiver to detect heart rate biosignal for a non-contact monitoring application. This work analysed antenna radiation parameters, beamwidth and polarisation to improve the radar characteristics and therefore remote heart rate detection on continuous waves at $2.4 \mathrm{GHz}$ [13]. Using four receivers improved the accuracy of detection in addition to the use of multiple frequencies $555-559 \mathrm{MHz}$, but the work did not use the antenna parameters for identification.

Studies have shown basic human movement can be detected through multiple antennas [14] and RFID (radio-frequency identification) tags [15]. For example, these systems were attached to multiple parts to isolate specific limb movement [15]. Antenna arrays are commonly used to detect people walking individually or in a group by using MIMO (multipleinput and multiple-outputs) sensors [16].

A large portion of open research in this area has used wireless channel characteristics, more for detecting movement than identifying and authenticating individuals. There is little evidence in published work to show that near-field antennas have been used to enhance security, in identifying and au- thenticating an individual. Preliminary results by the authors [17], on a number of people indicates that a wireless system could potentially be used to identify the individuals, however, techniques used need to be optimised for realtime applications, as targeted in this work.

For the first time, this work fully demonstrates the use of physical layer antenna characteristics to identify individual humans subjects at $2.45 \mathrm{GHz}$. The work combines antennas and propagation electromagnetic analysis at UHF (ultra high frequency) and microwave frequencies to identifying novel biometric features which are unique to the morphology of the person wearing the wireless communication device. Wearable antenna design could be exploited to give optimal resolution for a new biometric marker, for the purpose of identifying a unique signature for human subjects. Wearable antenna characteristics effects in close proximity (near-field) to a human body are discussed in Section II of this paper. Section III describes the standalone system circuitry design with component details. Section IV details the optimised analogue front end to utilise the hostile environment effects on the antenna and propagation characteristics, including a novel directional coupler simulation and prototype results. Experimental setup, measured results and analysis on fifty humans is presented in Section V. Section VI explains machine learning techniques implementation for feature distribution analysis, user identification on stationary and mobile human bodies, and thus, user identification on a longitudinal study.

\section{ANTENNA CHARACTERISTICS EFFECTS IN CLOSE PROXIMITY TO HUMAN BODY}

It is well understood that the major and minor fading characteristics of propagation channels react to varying body positions [18]. The human body attenuates electromagnetic waves from the antenna due to the lossy electrical properties of the tissue. This interaction can cause the antenna characteristics to change. From this interaction unique patterns or feature distributions related to an individual person are investigated. This paper introduces a novel technique to identify humans using the physical layer characteristics of the transceiver of a wearable device in close proximity to the human body. Whereas this detection is in the near-field of the antenna, the approach is much less sensitive to unwanted far-field environmental effects such as structures and multipath, as with the propagation channel measurements.

Wearable antennas are usually designed and optimised to minimise the variations in their characteristics when placed in the vicinity of the human body [19]. Human body effects on antenna and electromagnetic waves fundamentally depend on the electromagnetic interaction with the inhomogeneous dielectric tissue. Antenna type is one of the most impacting factors for mitigating the human effects on antenna characteristics. However, the design methodology required for this application, is to invert the requirements and investigate antennas which enhance and utilise coupling to the body. Fundamental antenna types such as microstrip fed patch, wire monopole, printed dipole, inverted $\mathrm{L}$ and circular loops have different impedances, excitation and radiation characteristics. 
Therefore, they can perform differently (e.g return loss) even when placed at the same location on the same human [20]. This variance is further compounded if the human changes their body position [21].

Proximity to tissue, its morphology and fluctuating electrical properties are the most impacting human factors on wearable antenna performance. Live tissue is largely inhomogeneous, differing in conductivity and permittivity that can vary with blood infiltration and hydration, which will alter the reactive nearfield E-field and H-Field components of an electromagnetic wave. Non-invasive measurements of these complex impedance properties (ratio of $\mathrm{E}$ and $\mathrm{H}$ fields) requires specialist equipment, such as bulky Vector Network Analysers (VNA). More localised measurements could be achieved by invasive techniques, using small in-vivo millimetre implantable devices [22]. Although, invasive procedures open new metrics in identifying unique characteristics, only non-invasive will be considered in this work. Herewith, surface worn antennas will be considered as this is more compatible with future security application requirements and technology adoption in next generation systems.

The electromagnetic coupling characteristics of an antennatissue interaction is unique to that individual, which theoretically makes it possible to identify individual humans by analysing antenna characteristic feature distribution. This human body interaction with an antenna changes the current distribution on the radiating element, which is dependent on the electromagnetic characteristics of the antenna, physical geometry, but also influenced by frequency, tissues dielectric properties, tissue morphology, movement and where an antenna is attached to a body [23]. This could be further enhanced by the use of several spatially located antennas, distributed on the surface of the body to increase the complexity of the secure authentication. Firstly, this work investigates the optimization of a single detection system.

\section{STANDALONE CIRCUITRY DESIGN}

VNAs do not provide a wearable, low-cost solution for the measurement of complex antenna characteristics. For practical purposes, wearable standalone circuitry has been designed and prototyped on (85 mm x $50 \mathrm{~mm}$ ) Rogers RO3010 $1.27 \mathrm{~mm}$ thick substrate for RF signal power measurement analysis, which is light enough to be worn by the user. The main function of the circuitry is to analyse impedance mismatch generated by a body-antenna interaction, which can be computed from the incident and reflected power. Using (1), the measured power can be related to return loss of incident and reflected excitation wave, at the antenna [24].

$$
R L(d B)=\operatorname{Pi}(d B m)-\operatorname{Pr}(d B m)
$$

The system block diagram is shown in Fig. 2, which includes the antenna (transmit/receive signals), directional coupler (signal coupler), VCO (signal generator), power detector (RF signal power to voltage convertor), microprocessor (analogue to digital converter) and an SD open log (data storage). An optimised design of the whole system block is implemented in the standalone circuit (Fig. 3) on a very small scale to measure return loss.

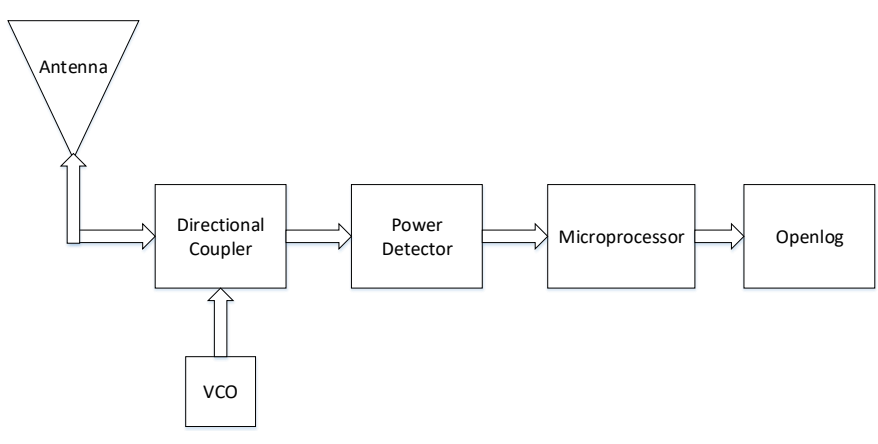

Fig. 2. Block system diagram of standalone detection circuit.

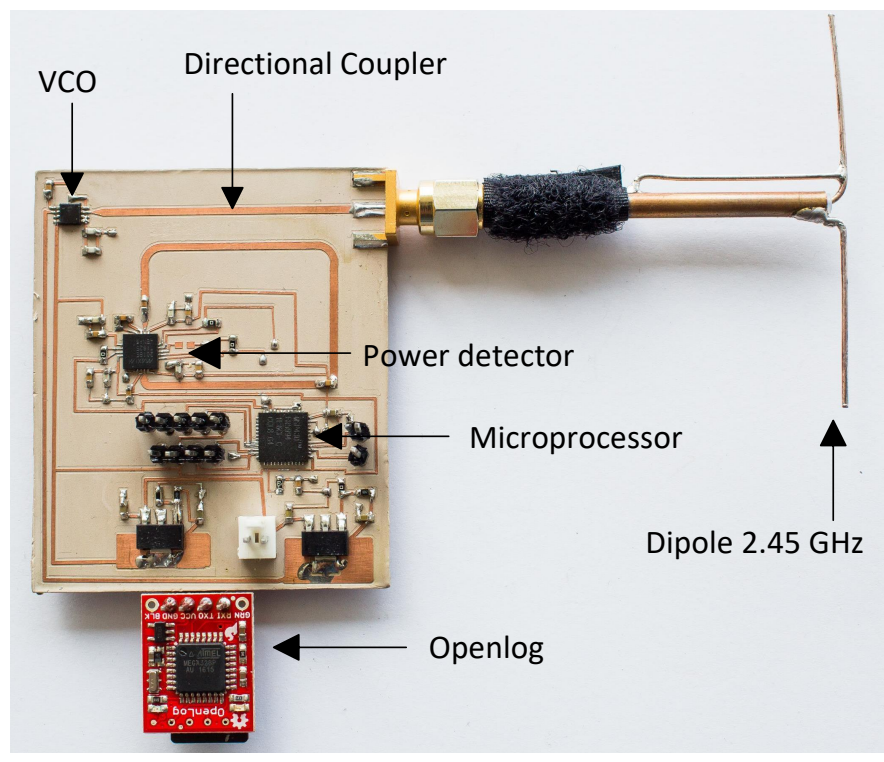

Fig. 3. Fabricated standalone circuit $(85 \mathrm{~mm} \times 50 \mathrm{~mm})$ with a $2.45 \mathrm{GHz}$ dipole antenna with $\frac{3}{4} \lambda$ Balun.

\section{A. Circuit Design}

The circuit prototype board is shown in Fig. 3. The VCO (MAX2750) generates a continuous signal of $-3 \mathrm{dBm}$ at 2.45 $\mathrm{GHz}$ which is input to the through transmission line (input port) of the directional coupler to the antenna (output port). A portion of signal on the through line is coupled to the power detector via the transmission line on coupled port and isolated port. Depending on the load impedance $\left(Z_{L}\right)$ of the antenna, which changes with each user because of their combination of tissue layers (tissue properties, muscle properties, differences in layer of skin, fat percentage and volume of tissues), a portion of the signal will be reflected power due to impedance mismatch. The RF reflected and incident power is converted to a linear scale voltage by the power detector (MAX2016) which is sent to a microprocessor (MSP430FR5994RGZ) for analysis and storage (Fig. 2). The power detector detection limits are from $-52 \mathrm{dBm}$ to $+10 \mathrm{dBm}$. The microprocessor is programmed to convert the analogue signal into 12-bit digital signal with 12-bit ADC and send it to the open log (DEV13712) to store it in an SD memory card automatically, in 30 second time windows. 
To meet the physical size requirements, the smallest surface mount passive components were selected. The circuit was designed from the system level diagram and Eagle PCB was used for the layout and track routing optimisation for $50 \Omega$ impedance characteristics.

\section{DIRECTIONAL COUPLER}

The directional couplers primary function is to provide incident and reflected power for return loss measurements. However, a novel directional coupler was designed to ensure maximum sensitivity to changes in the antenna load impedance. A coupled line directional coupler was selected for this application as it transmits power directly to an antenna and has a coupling coefficient selected to ensure minimum power levels can be detected.

\section{A. Coupled Line Directional Coupler Numerical Design}

The coupled line directional coupler consists of four ports (input, output, coupled and isolated), shown in Fig. 4. Coupling between ports can be calculated through the equations (2)-(4) [25].

$$
C=20 \log \frac{\left[1-c^{2} \cos ^{2} \beta l\right]^{1 / 2}}{c \sin \beta l}
$$

Where $C$ is coupling, $\beta$ is a propagation constant, $l$ is a length and voltage-coupling parameter $\mathrm{c}$ depends on even and odd impedance, $Z_{e}$ and $Z_{o}$, respectively [25].

$$
c=\frac{Z_{e}-Z_{o}}{Z_{e}+Z_{o}}
$$

For maximum coupling [25]:

$$
\beta l=\frac{\pi}{2}
$$

The directional coupler transmission lines were designed in Advanced Design System (ADS) to meet the $50 \Omega$ characteristic impedance of other RF components of the circuit on $1.27 \mathrm{~mm}$ thick, Rogers RO3010 substrate $(\tan \delta=0.017$, $\epsilon_{r}=10.2$ ). A detailed layout is presented in Fig. 4, with the key dimensions summarised in Table 1. A parameter sweep for the best combination of $Z_{e}$ and $Z_{o}$ (3) showed that the maximum range of power deviation at the coupling port is $Z_{e}$ $=51.85 \Omega$ and $Z_{o}=48.35 \Omega$ which gives an overall $Z o=$ $50 \Omega$. To ensure this, the spacing between the transmission lines is $4 \mathrm{~mm}$. The width of the transmission lines are 1.135 $\mathrm{mm}$ to maintain $50 \Omega$ impedance. Directional couplers provide maximum coupling by keeping both the transmission and coupled line length quarter of a wavelength $(30.60 \mathrm{~mm} / \sqrt{1} 0.2$ $=9.6 \mathrm{~mm}$ on Rogers RO3010 at $2.45 \mathrm{GHz}$ ). The length of the transmission line alters the phase of wave which was used to move the resonance to get minimum power levels at the coupled port (Fig. 5.) at $2.45 \mathrm{GHz}$. This provides a maximum power deviation at the coupling port due to an impedance mismatch at the output port through the body-antenna interaction. The length of transmission and coupled lines are 22 $\mathrm{mm}$, by adding $4 \mathrm{~mm}$ on each side of transmission lines for the non-interference connections to other components leaves a
TABLE I

Coupled Line Directional Coupler Dimensions $(2.45 \mathrm{GHz})$

\begin{tabular}{cccccc}
\hline \multicolumn{6}{c}{ Dimensions $(\mathrm{mm})$. refer to Fig. 4 } \\
\hline L1 & L2 & L3 & H1 & H2 & A(deg) \\
\hline 22.00 & 4.00 & 4.00 & 1.13 & 1.16 & $90^{\circ}$ \\
\hline
\end{tabular}

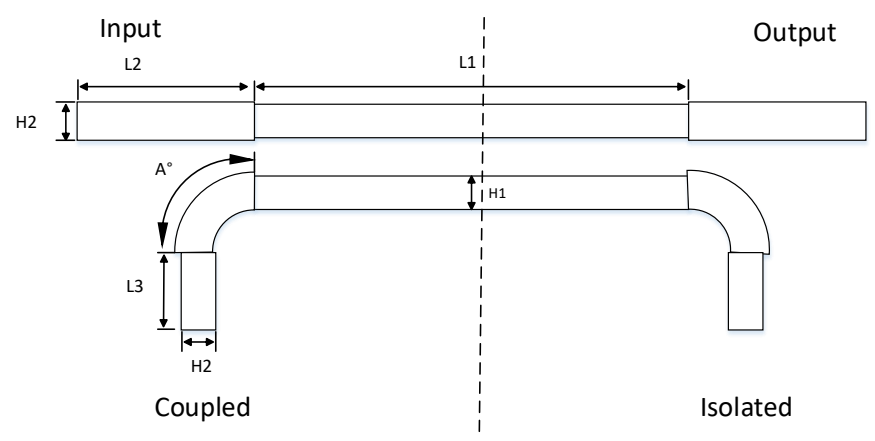

Fig. 4. Directional coupler symmetrical design.

total track length of approximately three quarter wavelengths (30 mm) on the RO3010 substrate. Theoretical coupling for quarter wavelength directional couplers are usually between 8$10 \mathrm{~dB}$. Through ADS analysis, this three-quarter wavelength directional coupler has a coupling coefficient $C$ (2) of 29.14 dB.

Table 2 shows nine different EM simulation results of layout in ADS by changing load at output port of directional coupler. Results show the power level's at input port, coupled port and isolated port by changing load at output port (antenna). The simulation results show that this directional coupler provides up to $25 \mathrm{~dB}$ variance in the detection range at the coupling port by altering different loads at the output port, which is advantageous for human identification.

\section{B. Fabricated Coupled Line Directional Coupler Experimen- tal Analysis}

The fabricated directional coupler is shown in Fig. 6. To isolate the performance of the direction coupler it was analysed using a four port Rhode \& Schwarz ZVB VNA, before integration with systems circuitry shown in Fig. 3, The results for each of the three directional coupler ports are shown in Fig. 5 , with a perfectly matched $50 \Omega$ load attached to output port.

TABLE II

Em Simulation ImPedance Mismatch Results For the AdS LAYOUT With DIFFERENT LOADS AT THE OUTPUT PORT $(2.45 \mathrm{GHz})$

\begin{tabular}{lllll}
\hline $\begin{array}{l}\text { Load at output } \\
\text { Port }(\Omega)\end{array}$ & $\begin{array}{l}\text { Input } \\
\text { port }(\mathrm{dB})\end{array}$ & $\begin{array}{l}\text { Coupled } \\
\text { port }(\mathrm{dB})\end{array}$ & $\begin{array}{l}\text { Isolated } \\
\text { port }(\mathrm{dB})\end{array}$ & $\begin{array}{l}\text { Output } \\
\text { port }(\mathrm{dB})\end{array}$ \\
\hline 50 & -27 & -50 & -22 & 0 \\
10 & -3.5 & -25 & -23 & -3 \\
20 & -7 & -29 & -23 & -1 \\
30 & -11 & -33 & -22 & -0.5 \\
40 & -19 & -39 & -21 & -0.1 \\
60 & -25 & -45 & -21 & 0 \\
70 & -17 & -38 & -22 & 0 \\
80 & -14 & -35 & -22 & 0 \\
500 & -2 & -24 & -22 & -5 \\
\hline
\end{tabular}




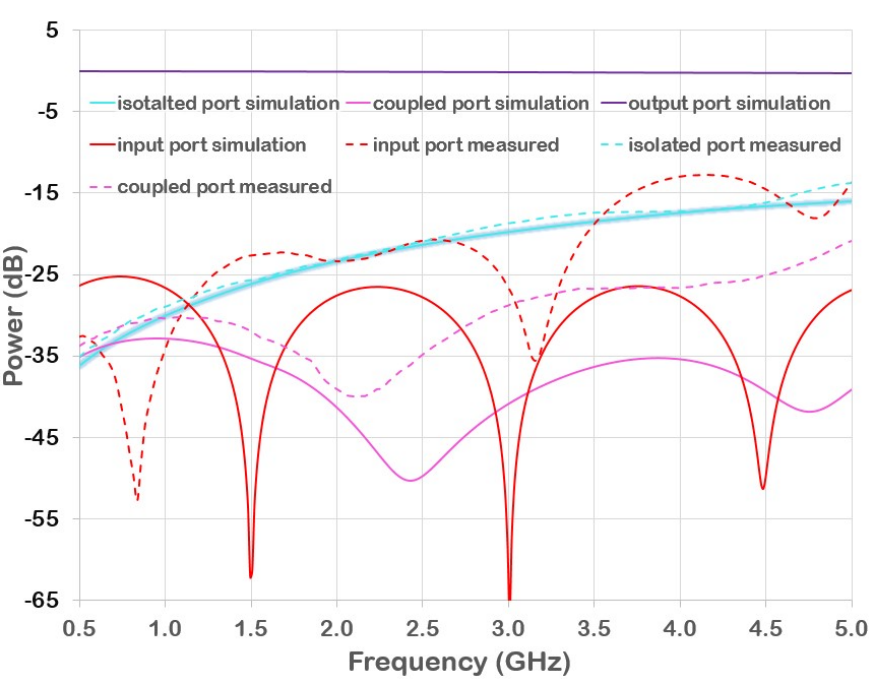

Fig. 5. ADS layout EM simulation results for $50 \Omega$ and a measured directional coupler 3 ports analysis with an output port terminated with a $50 \Omega$ matched load.

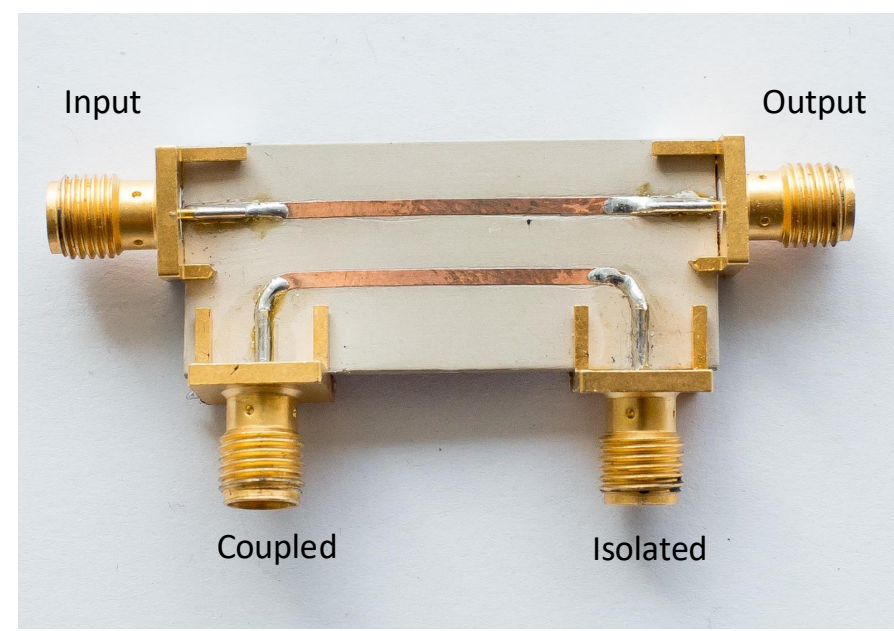

Fig. 6. Fabricated $\frac{3}{4} \lambda$ directional coupler on Rogers RO3010 substrate.

Fig. 5 shows that a slight frequency offset in the input and the coupling port minimum, with a less prominent null, moved from $-50 \mathrm{~dB}$ to $-40 \mathrm{~dB}$ in the coupling port and from $-62 \mathrm{~dB}$ to $-54 \mathrm{~dB}$ in the input port, as compared to EM simulation results. This may be attributed to the SMA connectors used in the measured case. The isolated port stays almost the same as in the EM simulation results.

Verification experiments were conducted on two body parts of two human subjects at $2.45 \mathrm{GHz}$. A $2.45 \mathrm{GHz}$ dipole antenna was connected to output port of the directional coupler. The coupled port and isolated port results are shown in Table 3. Results show up to $21 \mathrm{~dB}$ range of power deviation at the coupled port with the body-antenna interaction, which is good for feature analysis, but also in agreement with $25 \mathrm{~dB}$ variations in simulated results.
TABLE III

FABRICATED DIRECTIONAL COUPLER EXPERIMENTAL RESULTS ON A VNA FOR DIFFERENT SITUATIONS

\begin{tabular}{lll}
\hline Situation & Coupled Port & Isolated Port \\
\hline No antenna & -21.35 & -21.16 \\
With Antenna & -36.41 & -21.37 \\
$50 \Omega$ load & -37.05 & -22.39 \\
$1^{\text {st } \text { subject arm on antenna }}$ & -37.22 & -22.17 \\
$2^{\text {nd } \text { subject arm on antenna }}$ & -42.10 & -22.11 \\
$3^{\text {rd } \text { subject arm on antenna }}$ & -29.03 & -21.11 \\
$4^{\text {rth } \text { subject arm on antenna }}$ & -32.40 & -21.26 \\
$1^{\text {st } \text { subject chest on antenna }}$ & -33.53 & -21.90 \\
$2^{\text {nd }}$ subject chest on antenna & -39.89 & -21.76 \\
$3^{\text {rd }}$ subject chest on antenna & -34.07 & -21.82 \\
$4^{\text {rth }}$ subject chest on antenna & -33.18 & -22.26 \\
\hline
\end{tabular}

\section{EXPERIMENTAL SETUP AND MEASUREMENT}

A perspex test-bed was fabricated to protect the prototyped circuit and to aid efficient and repeatable measurement of multiple users. The perspex sheet has a window cut at the antenna, which exposes the antenna to the human tissue at a fixed distance of $10 \mathrm{~mm}$ for initial identification measurements.

\section{A. Return loss measurement for human identification}

A $2.45 \mathrm{GHz}$ dipole antenna was configured on the standalone detection circuitry and used for return loss measurement on the wrists and triceps of fifty human subjects at room temperature $\left(21-25{ }^{\circ} \mathrm{C}\right)$ as shown in Fig. 7. Dipole antennas at $2.45 \mathrm{GHz}$ provide a relatively dynamic return loss when mounted on different tissue, yet can maintain the impedance bandwidth at the $2.45 \mathrm{GHz}$ frequency band. As such, it has been selected in this work to demonstrate the human identification concept. Each identification measurement was repeated four times for both body parts, which includes repositioning the body part between measurements. Each measurement was taken for thirty seconds and during that time period the user and circuitry were stationary. The measured results presented in Fig. 8 shows a graph of power against time which includes the coupled port, the isolated port and the return loss measurements with continuous waves at $2.45 \mathrm{GHz}$ for thirty seconds for one person. The coupled port power level is relatively low which makes it sensitive to channel noise (WIFI normally operates at $2.45 \mathrm{GHz}$ ) and creates non-periodic pulses. By taking the root mean square (RMS) of the power detector values (Fig. 9.), it is visually possible to correlate and differentiate some of the test subjects. The non-periodic pulses are less then $0.3 \%$ and does not significantly effect RMS of return loss. Fig. 9. shows that each body part interacts differently with the antenna, and therefore there is potential for feature correlation and detection. The coupled port values of the wrist for the subjects falls between $-33.5 \mathrm{dBm}$ to $-41.8 \mathrm{dBm}$ and the triceps fall between -34.2 $\mathrm{dBm}$ to $-47 \mathrm{dBm}$. In a comparison between body parts, it shows that the triceps can provide better human identification than a wrist as it varies more than the wrist for different subjects. However, visual or modal identification is limited due to the simplicity of the feature selection algorithm. In this scenario there is not enough range $(8 \mathrm{dBm})$ in wrist measurements to identify all fifty people. By including multiple 

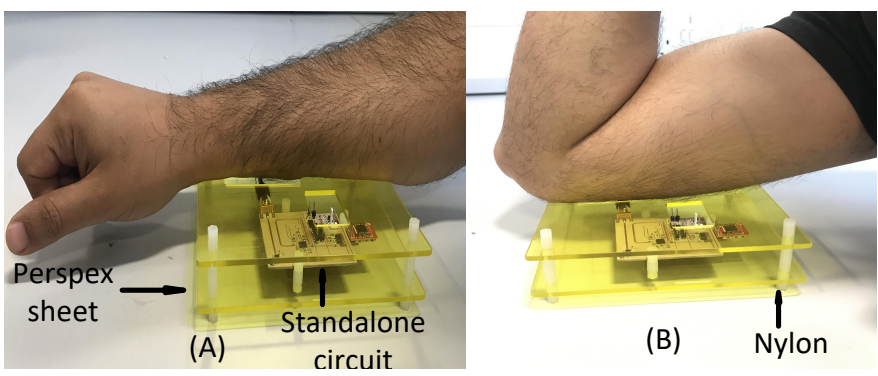

Fig. 7. Hardware test-bed and body positions for measurements: (a) wrist (b) tricep.

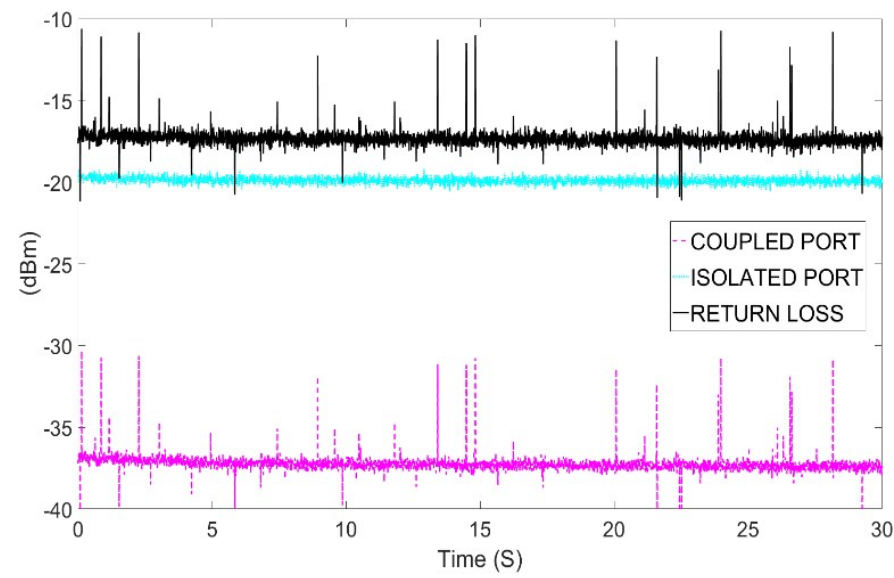

Fig. 8. An example of power detector output as a function of time, from each port on the directional coupler: coupled port; isolated port; and calculated return loss.

body parts in the identification, a larger number of humans would be possible. However, on a practical application this would require multiple trusted networked systems, correlated and communicating together. Therefore this work looks at the potential feature identification from a single body part. To investigate the number of potential subjects which can be identified from single body part feature correlation, a more complex identification algorithm was introduced, based on machine learning techniques.

\section{HUMAN IDENTIFICATION USING MACHINE LEARNING TECHNIQUES}

In this section, machine learning is used as an approach for identifying the person by considering only the antenna return loss feature of the wrist (single body part) of fifty subjects. This work is not focused on developing the most optimal or efficient mathematical feature recognition system, but rather uses this machine learning approach to demonstrate the biometric concept capability.

\section{A. Machine Learning Feature Extraction}

A feature extraction based on the Inverse Discrete Fourier Transform (iDFT) of the return loss was calculated. The iDFT, $z(m)$ is computed with fast Fourier transform (FFT) algorithm $x(n)$ and is calculated as [26],

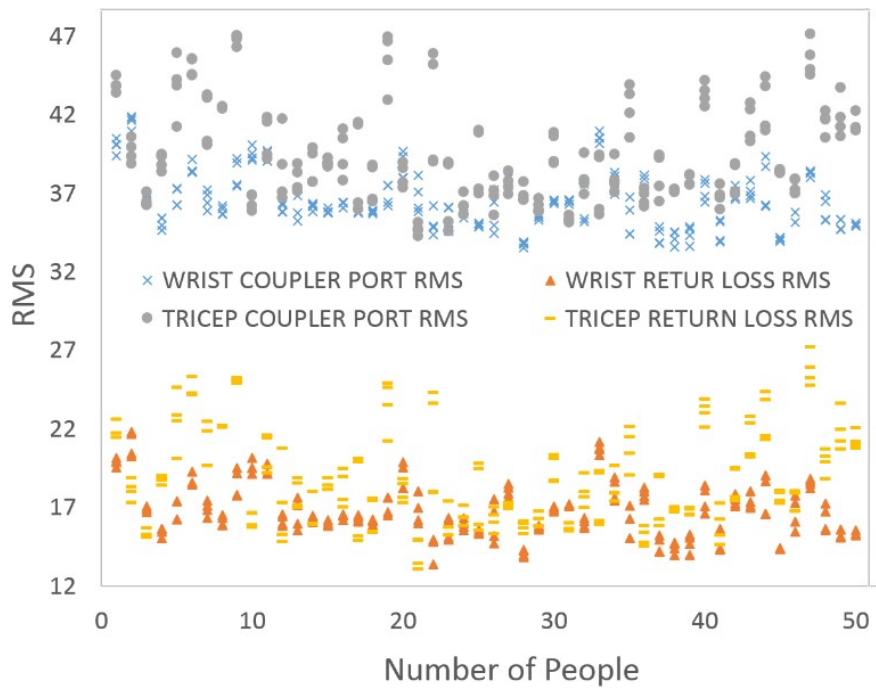

Fig. 9. Wrist and triceps measurement results on fifty human subjects.

$$
z(j)=\frac{1}{N} \sum_{n=1}^{N} x(n) L_{N}^{-(n-1)(j-1)}
$$

where, $x(n)$ is FFT of the return loss, $n=1,2, \ldots, N$ number of data points of return loss, $j$ is the number of return loss iterations, and $L_{N}$ is the return loss expressed as, $L_{N}=e^{(-2 \pi i) / N}$

In inverse FFT, an $N$-element vector $l$ of return loss is conjugate symmetric when the $i^{\text {th }}$ element satisfies $l(i)=$ $\operatorname{conj}(l(N-i+1, N+1))$ for each element of $l$ [27].

Linear discriminant analysis (LDA) is then used as a linear supervised feature-projection that evaluates a linear matrix from inverse DFT feature vector to form the between-class scatter large and the within-class scatter small [28]. The LDA was implemented to find a coordinate system to maximize the class separability for the projected features. It is expressed as $y=W^{T} z$, where $z$ is the feature vector with $n$ dimensionality, $y$ is the projected feature vector with $k$ dimensionality $(k \leq$ $n$ ), and $S$ is an $n \times k$ matrix. The LDA is primarily created on a family of functions of scatter matrices. The within-class scatter matrix is calculated as [29],

$$
S_{W}=\sum_{c=1}^{K} \sum_{i=1}^{M} r_{i}^{(c)}\left(z_{i}-m^{(c)}\right)\left(z_{i}-m^{(c)}\right)^{T}
$$

where $z_{i}, i=1, \ldots, M$ denotes the $n$-dimensional feature vectors, $M$ is the number of features, $m^{(c)}$ is the mean vector for class $c$, and $r_{i}^{(c)} i=1$ if $z_{i} \in c$ and 0 otherwise. Further, the between-class scatter matrix is calculated as [29],

$$
S_{B}=\sum_{c=1}^{K} N_{c}\left(z_{i}-m^{(c)}\right)\left(z_{i}-m^{(c)}\right)^{T}
$$

where $m$ is the mean vector for all features. Finally, the total scatter matrix is the covariance for all evaluated features, irrespective of the class [29] and is calculated as, 


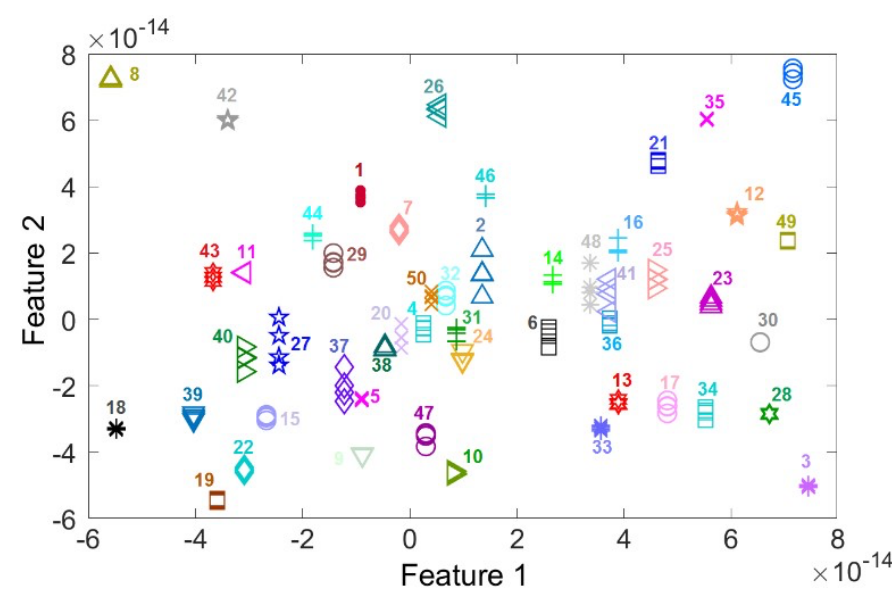

Fig. 10. Projected features from LDA for different subjects.

$$
S_{T}=\sum_{i=1}^{M}\left(z_{i}-m\right)\left(z_{i}-m\right)^{T}=S_{W}+S_{B}
$$

For the total scatter matrix, the distributed measure is the determinant. Thus, an $n \times k$ matrix $W$ is calculated using Fisher's method that maximizes the learning criterion [29],

$$
\frac{\operatorname{det}\left(W^{T} S_{B} W\right)}{\operatorname{det}\left(W^{T} S_{W} W\right)}
$$

The matrix $W$ is composed of $k$ eigenvectors corresponding to the $k$ largest eigenvalues of $S_{W}^{(-1)} S_{B}$. Since the betweenclass scatter matrix $S_{B}$ has a maximum rank of $K-1$, the value of $k$ must be stated less than $K$ [28]. Thus, the dimensionality of the projected feature space is limited by the number of classes. To endure with this limitation, the total scatter matrix is used instead of the between-class scatter matrix in the learning criterion, because the dimensionality of the projected features is not limited by the number of classes [28]. Thus, from this result, the $k$-dimensional feature $y$ is obtained as shown in Fig. 10, illustrating clusters for each of the test subjects with different color and shapes as projected by LDA.

After the feature projection, the reduced features are given to a multi-layer perceptron (MLP) classifier. The eight outputs of the LDA are used to construct the input layer of the MLP. The weights of the network are computed by the backpropagation algorithm with three hidden layers consisting seventy-two neurons and fifty neurons in output layer to recognize fifty classes. This network structure was determined by trial and error. The selection criterion was based on the convergence of the learning error from different combinations [30]. While testing the performance, the projected features from LDA is given to the trained MLP classifier which gives high output from the respective neurons in the output layer. Thus, one set of return loss data from each subject was tested on the trained MLP and the overall classification accuracy was evaluated as the number of correct data assessments divided by the total number of data assessments, which results in $98.90 \%$.

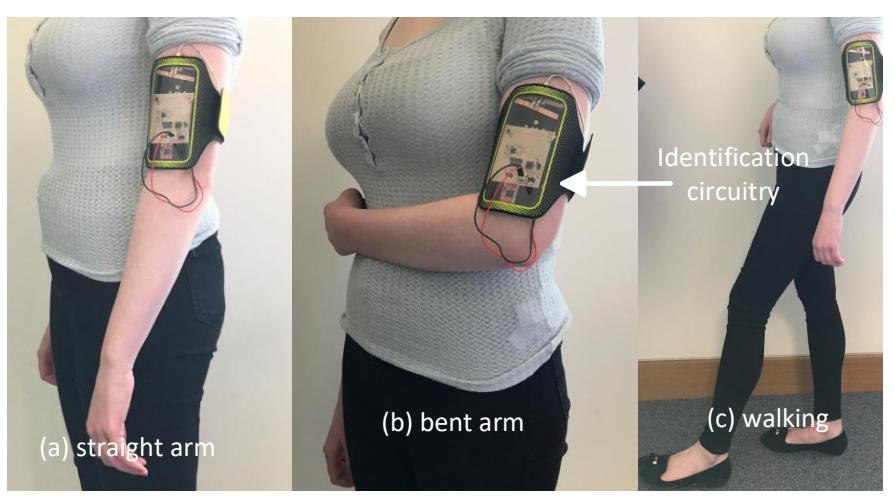

Fig. 11. Dynamic body positions with armband mounted system (a) stationary straight arm (b) stationary bent arm (c) walking.

TABLE IV

CLASSIFICATION RESULTS ON DIFFERENT TIME FRAME WINDOWS

\begin{tabular}{llll}
\hline $\begin{array}{l}\text { Sr } \\
\text { No }\end{array}$ & $\begin{array}{l}\text { Time frame } \\
(\mathrm{sec})\end{array}$ & $\begin{array}{l}\text { Window size } \\
(\text { samples })\end{array}$ & $\begin{array}{l}\text { Accuracy } \\
(\%)\end{array}$ \\
\hline 1 & 1 & 160 & 46.29 \\
2 & 2 & 320 & 71.35 \\
3 & 3 & 480 & 78.62 \\
4 & 5 & 800 & 85.51 \\
5 & 10 & 1600 & 88.04 \\
6 & 20 & 3200 & 90.59 \\
7 & 30 & 4800 & 93.16 \\
\hline
\end{tabular}

Although the classification is sufficient for identification, the algorithm could be further enhanced with the addition of a third feature.

\section{B. Human Identification Based on Body Movements}

Human identification was investigated on twenty subjects completing different body posture movements: straight arm, bent arm and walking as shown in Fig. 11. The same configuration as used in Section III was fitted in an arm band and attached to the tricep of each subject. Two measurements were conducted for each position for a duration of 30 seconds.

The evaluated antenna return loss for each of the three body movements was considered in varying time frame windows. Firstly, to extract key points from the return loss, $3^{\text {rd }}$ order Auto-regression (AR) models with root sum of square (RSS) level and time-domain features were used to build a feature vector. The AR model states each return loss sample as a linear combination of previous samples $x_{t-1}$ plus a white noise error term $w(t)$ [31], [32]. The AR coefficients are calculated as,

$$
A R(t)=\sum_{p=1}^{P} a_{p} x(t-p)+w(t)
$$

where $x(t)$ is the $t^{t h}$ sample return loss, $a_{p}$ is each of AR coefficients, $w(t)$ is the white error noise term and $P$ is the order of the AR model ( 3 in this case). After fitting the auto-regressive model, the AR coefficients are used as part of the feature vector with root sum of absolute square level [33] calculated as, 


$$
R S S=\sqrt{\sum_{t=1}^{N}\left|x_{t}\right|^{2}}
$$

where $N$ denotes the length of the sample return loss.

Thus, an $N$-dimensional return loss signal is represented with four-point feature vector. Further, an LDA feature projection is used to spread the between-class scatter from evaluated feature points [28]. The projected 20 class features are given to a new MLP classifier. The input layer of the MLP is constructed with 4 neurons from LDA. The feed-forward algorithm with three hidden layers consisting of 44 neurons is used to evaluate the weights of the network. Further, the output layer has 20 neurons to identify 20 classes (Humans). This network structure was determined by trial and error. For training the network, from each set of return loss measurements, 3840 samples $(80 \%)$ were used for training session. The performance of the system is tested on the remaining 960 (20\%) return loss samples from each set. The feature vectors are calculated from these samples followed for the projected feature vector. This projection is passed through the trained MLP classifier which gives a high output from the respected neuron in an output layer. Thus, for testing the performance of system, different time frame windows are being considered and the classification accuracy is being evaluated as shown in Table IV. The time frame window for $1 \mathrm{sec}$ shows the classification accuracy of $46.29 \%$ and further incrementing the window time frame width to $2 \mathrm{sec}$, substantialy increases the accuracy to $71.35 \%$. Concurrently, while increasing the time-frame, the classification accuracy increased gradually and best results were obtained while using a complete time-frame window of $30 \mathrm{sec}$ to be $93.16 \%$.

\section{Human Identification based on various day-to-day routine experimentation}

An experiment was conducted on four people for over a period of one month to observe the classification accuracy for potential changes in daily tissue properties. To observe the longitudinal effect of change in tissue, the measurements samples are taken for five consecutive days. After day 5, measurements are taken once per week for up to four weeks. Training data was taken on day 1 only, which included four measurement samples. Based on five weeks, which was a total of 12 measurements on different days, the training data accounted for $33.33 \%$ data and the remaining $66.66 \%$ of the data was analysed for identification. This biometric concept is targeted at close fitting wearable devices, where the spacing between the antenna and the tissue is controlled using a wearable armband (Fig. 11). Controlling the antenna body spacing is important for maintaining the performance on most wireless wearable devices that do not use large microstrip type antennas. The experimental measurements therefore include practical errors associated with removing and replacing the antennas over multiple days. The projection features graph for this system configuration, where each colour represents a different person and each shape represents a different day and a week is shown in Fig. 12. Despite the potential inaccuracies in

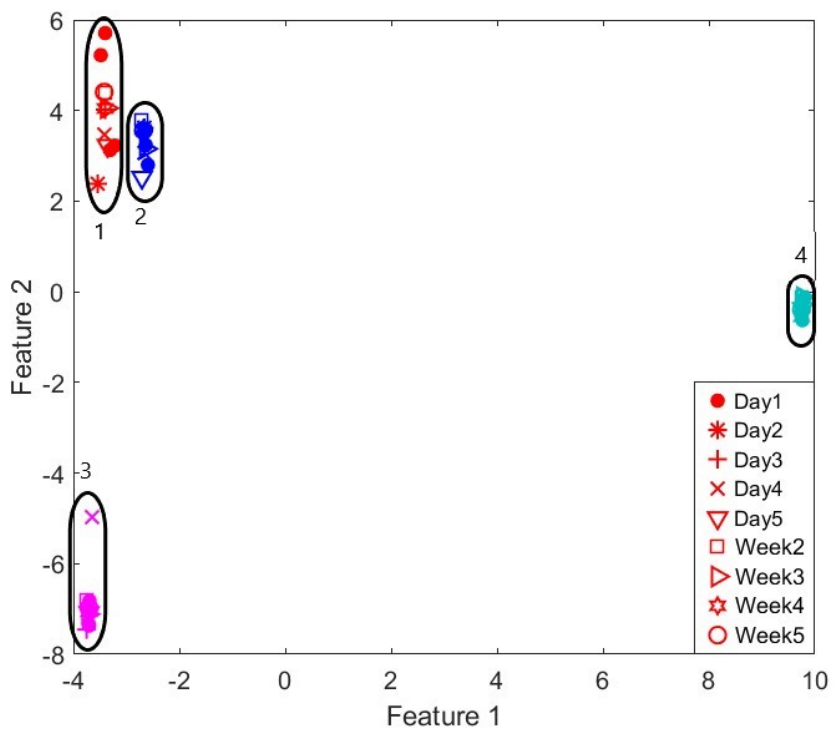

Fig. 12. Projected features based on different test subjects (numbered 1-4) for various days data.

placement, spacing, a classification identification accuracy of $97.38 \%$ was achieved with day 1 samples as the training data with the same system configuration of iDFT feature extraction with LDA feature projection and MLP classification.

The results show that there is some divergence in the practical results with time, in particular person 1, due to compounded potential errors such as tissue property changes, loss/gain of weight, placement measurement errors. Nonetheless, it is still possible to identify each subject.

\section{CONCLUSION}

This work shows that individuals can be identified using wearable antenna performance for both stationary and dynamic movements with classification accuracies of up to 98\%. Standalone circuit is demonstrated which can measure antenna characteristics in real time and identify individuals without the need for additional bulky physical layer sensing hardware. A directional coupler is the main additional integrated component in the physical layer circuitry, which could be commercially packaged using surface mount components. A novel directional coupler of three quarter wavelengths, prototyped on Rogers RO3010 has been introduced in this paper which enhances the human identification. The work demonstrates that the human body causes unique attenuation in antenna characteristics which reflects the uniqueness of each subject tissue morphology. The return loss from the wrist of each subject in stationary state was tested on the trained iDFT based feature classifier and overall classification accuracy was found to be $98.60 \%$. Moreover, for mobile human bodies, each subjects data was tested on time-domain based feature trained classifier models and the accuracy was found to be $93.16 \%$ on a 30 second time frame window. One months data was used to demonstrate the research concept, however, further work could include an extensive engineering measurement campaign to capture abnormal variations in humans and more complex 
algorithms. The variance of the Human body could also be captured as a standalone feature to enhance the authentication and security of the biometric concept. For example, a change in tissue properties by gaining weight, skin changes, muscle development or any other iteration in the long term would be unique to that individual, and updated periodically to the algorithm. An exciting area to explore in future work is if there are novel optimal antenna solutions, which could enhance identification as well as communication. The testsubjects could be divided into similar feature sets, such as: sex, ethnic origin, weight, height or age, etc. which would be a challenging practical test scenario. In-situ repetitive measurements could be performed in an extensive measurement campaign to determine the variability in feature correlation for secure key generation, which, given the dynamic nature of the body, would require generation of some partial polynomial fits of the feature co-efficient(s). Overall, the wireless physical layer biometric capability presented in this work could be combined with other biometrics or multiple body parts to enhance the uniqueness, and robustness of the user signature or alternatively, merged with other secure techniques to enhance the security of next generation user systems in wearable environments.

\section{REFERENCES}

[1] Z. Guo, N. Karimian, M. M. Tehranipoor, and D. Forte, "Hardware security meets biometrics for the age of IoT," in 2016 IEEE International Symposium on Circuits and Systems (ISCAS), May 2016, pp. 1318-1321.

[2] M. Gowtham and S. S. Ahila, "Privacy enhanced data communication protocol for wireless body area network," in 2017 4th International Conference on Advanced Computing and Communication Systems (ICACCS), Jan. 2017, pp. 1-5.

[3] S. Ergin, A. K. Uysal, E. S. Gunal, S. Gunal, and M. B. Gulmezoglu, "ECG based biometric authentication using ensemble of features," in 2014 9th Iberian Conference on Information Systems and Technologies (CISTI), Jun. 2014, pp. 1-6.

[4] Q. Xu, Y. Chen, B. Wang, and K. J. R. Liu, "Radio Biometrics: Human Recognition Through a Wall," IEEE Transactions on Information Forensics and Security, vol. 12, no. 5, pp. 1141-1155, May 2017.

[5] X. Duan and X. Wang, "Authentication handover and privacy protection in $5 \mathrm{~g}$ hetnets using software-defined networking," IEEE Communications Magazine, vol. 53, no. 4, pp. 28-35, Apr. 2015.

[6] N. H. M. Rais, P. J. Soh, F. Malek, S. Ahmad, N. B. M. Hashim, and P. S. Hall, "A review of wearable antenna," in 2009 Loughborough Antennas Propagation Conference, Nov. 2009, pp. 225-228.

[7] P. Schneider and G. Horn, "Towards 5g Security," in 2015 IEEE Trustcom/BigDataSE/ISPA, vol. 1, Aug. 2015, pp. 1165-1170.

[8] K. Diederichs, A. Qiu, and G. Shaker, "Wireless Biometric Individual Identification Utilizing Millimeter Waves," IEEE Sensors Letters, vol. 1, no. 1, pp. 1-4, Feb. 2017.

[9] M. Mohamed and M. Cheffena, "Received Signal Strength Based Gait Authentication," IEEE Sensors Journal, vol. 18, no. 16, pp. 6727-6734, Aug. 2018.

[10] N. Zhao, Z. Zhang, M. U. Rehman, A. Ren, X. Yang, J. Zhao, W. Zhao, and B. Dong, "Authentication in Millimeter-Wave BodyCentric Networks Through Wireless Channel Characterization," IEEE Transactions on Antennas and Propagation, vol. 65, no. 12, pp. 66166623, Dec. 2017.

[11] X. Yang, S. A. Shah, A. Ren, N. Zhao, D. Fan, F. Hu, M. U. Rehman, K. M. vonDeneen, and J. Tian, "Wandering Pattern Sensing at S-Band," IEEE Journal of Biomedical and Health Informatics, vol. 22, no. 6, pp. 1863-1870, Nov. 2018.

[12] Y. Li, D. Xue, E. Forrister, G. Lee, B. Garner, and Y. Kim, "Human Activity Classification Based on Dynamic Time Warping of an OnBody Creeping Wave Signal," IEEE Transactions on Antennas and Propagation, vol. 64, no. 11, pp. 4901-4905, Nov. 2016.
[13] M. Nosrati and N. Tavassolian, "Effects of Antenna Characteristics on the Performance of Heart Rate Monitoring Radar Systems," IEEE Transactions on Antennas and Propagation, vol. 65, no. 6, pp. 32963301, Jun. 2017.

[14] K. Shin, K. Yabata, K. Momota, and M. Nishi, "Evaluation of the human detection system using UHF band TV waves for the car security," in 2016 International Symposium on Antennas and Propagation (ISAP), Oct. 2016, pp. 222-223.

[15] S. Amendola, L. Bianchi, and G. Marrocco, "Movement Detection of Human Body Segments: Passive radio-frequency identification and machine-learning technologies." IEEE Antennas and Propagation Magazine, vol. 57, no. 3, pp. 23-37, Jun. 2015.

[16] K. Sato, K. Nishimori, N. Honma, and H. Makino, "Basic study on human detection by MIMO sensor with antennas at ceiling and floor," in 2016 International Symposium on Antennas and Propagation (ISAP), Oct. 2016, pp. 500-501.

[17] W. Saadat, S. Raurale, G. Conway, and J. McAllister, "User Identification Through Wearable Antenna Characteristics at $2.45 \mathrm{GHz}, 2018$.

[18] G. A. Conway, S. L. Cotton, and W. G. Scanlon, "An Antennas and Propagation Approach to Improving Physical Layer Performance in Wireless Body Area Networks," IEEE Journal on Selected Areas in Communications, vol. 27, no. 1, pp. 27-36, Jan. 2009.

[19] R. B. V. B. Simorangkir, A. Kiourti, and K. P. Esselle, "UWB Wearable Antenna With a Full Ground Plane Based on PDMS-Embedded Conductive Fabric," IEEE Antennas and Wireless Propagation Letters, vol. 17, no. 3, pp. 493-496, Mar. 2018.

[20] M. M. Khan, S. Shanaz, and Abdullah-Al-Mamun, "Investigation of performance parameters of different wearable narrowband antennas in close proximity to the human body," in 2012 15th International Conference on Computer and Information Technology (ICCIT), Dec 2012, pp. 627-631.

[21] F. Mani and R. D'Errico, "Macro- and micro-diversity in indoor bodyto-body channels," in 2016 10th European Conference on Antennas and Propagation (EuCAP), Apr. 2016, pp. 1-5.

[22] D. Celinskis and B. C. Towe, "Characterization of the implantable neurostimulator-based wireless bioimpedance measurement technique,' in 2016 IEEE EMBS International Student Conference (ISC), May 2016, pp. $1-4$.

[23] P. Li, L. Zhang, F. Liu, and J. Amely-Velez, "Optimized matching of an implantable medical device antenna in different tissue medium using load pull measurements," in 2015 86th ARFTG Microwave Measurement Conference, Dec. 2015, pp. 1-4.

[24] D. M. Pozar, Microwave engineering, 4th ed. Hoboken, NJ: Wiley, 2012, oCLC: ocn714728044.

[25] R. E. Collin, Foundations for microwave engineering, 2nd ed., ser. IEEE Press series on electromagnetic wave theory. New York: IEEE Press, 2001.

[26] L. Cimini, "Analysis and Simulation of a Digital Mobile Channel Using Orthogonal Frequency Division Multiplexing," IEEE Transactions on Communications, vol. 33, no. 7, pp. 665-675, Jul. 1985.

[27] F. Zhou, J. F. Feng, and Q. Y. Shi, "Texture feature based on local Fourier transform," in Proceedings 2001 International Conference on Image Processing (Cat. No.01CH37205), vol. 2, Oct. 2001, pp. 610613 vol.2.

[28] B. Prasad and S. R. M. Prasanna, Speech, Audio, Image and Biomedical Signal Processing using Neural Networks. Springer, Dec. 2007, googleBooks-ID: YxdqCQAAQBAJ.

[29] J. Chu, I. Moon, Y. Lee, S. Kim, and M. Mun, "A Supervised FeatureProjection-Based Real-Time EMG Pattern Recognition for Multifunction Myoelectric Hand Control," IEEE/ASME Transactions on Mechatronics, vol. 12, no. 3, pp. 282-290, Jun. 2007.

[30] J. Mao and A. K. Jain, "Artificial neural networks for feature extraction and multivariate data projection," IEEE Transactions on Neural Networks, vol. 6, no. 2, pp. 296-317, Mar. 1995.

[31] Y. Grenier, "Time-dependent ARMA modeling of nonstationary signals," IEEE Transactions on Acoustics, Speech, and Signal Processing, vol. 31, no. 4, pp. 899-911, Aug. 1983.

[32] S. L. Cotton, G. A. Conway, and W. G. Scanlon, "A Time-Domain Approach to the Analysis and Modeling of On-Body Propagation Characteristics Using Synchronized Measurements at $2.45 \mathrm{GHz}$,' IEEE Transactions on Antennas and Propagation, vol. 57, no. 4, pp. 943-955, Apr. 2009

[33] N. G. Paulter, D. R. Larson, and J. J. Blair, "The IEEE standard on transitions, pulses, and related waveforms, Std-181-2003," IEEE Transactions on Instrumentation and Measurement, vol. 53, no. 4, pp. 1209-1217, Aug. 2004. 


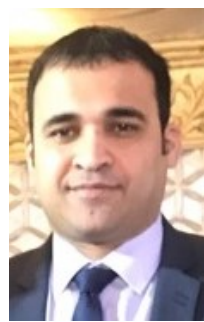

Waqar Saadat (S'15) (S15) received the B.Sc. degree in Computer Engineering from the University of Engineering and Technology, Taxila, Pakistan., in 2007, and the M.Sc. degree in Electrical and Electronics Engineering from the University of Glasgow, Glasgow, U.K., in 2008. He is currently pursuing the $\mathrm{Ph} . \mathrm{D}$. degree in Electrical and Electronics Engineering with the Queens University Belfast, Belfast, U.K. His research interests include physical layer security for wireless communication and antenna design in wearable applications.

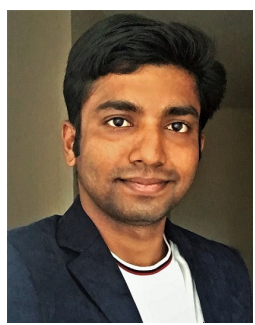

Sumit A. Raurale (S14) received the B.E. degree in Electronics Design Technology from Shri Ramdeobaba College of Engineering and Management Nagpur, India, in 2012 and the M.Tech. degree in Electronic Systems and Communication from Government College of Engineering Amravati, India, in 2014. In 2015, he was a Junior Research Fellow with the Visvesvaraya National Institute of Technology, Nagpur, India. He is currently pursuing the Ph.D. degree in Electrical and Electronic Engineering at Queens University Belfast, U.K. His research interest includes Biomedical and RF signal processing, Human-Computer interface and Machine learning.

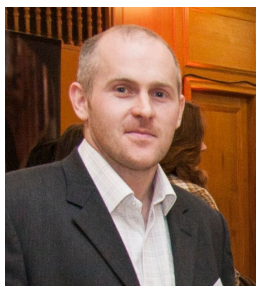

Gareth A. Conway received a BEng Hons. Degree in Electronic Systems from the University of U1ster, UK in 2004. In 2008, he completed a Ph.D. degree in Electronic Engineering, entitled Wearable Antennas for On-Body Wireless Communications at Queens University of Belfast, (UK). On completion of his doctorate, he spent three years as a commercial research engineer, specializing in antennas and propagation for mobile communication. In 2011, he rejoined QUB to complete an EPSRC Knowledge Transfer Secondment with Toumaz Healthcare Ltd., undertaking research and development in Innovative body-worn antennas for medical devices. In 2013 he became a Lecturer in Communications Engineering at ECIT, Queens University of Belfast. Dr. Conway has authored or co-authored over 40 international conference and journal papers. His research interests include antennas, human tissue equivalent materials, wave propagation and computational electromagnetism for wearable and implantable communications.

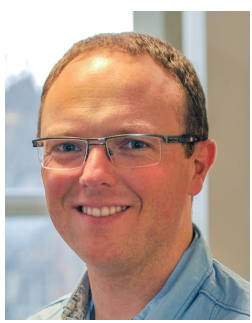

John McAllister (S02-M2004-SM12) received the $\mathrm{Ph} . \mathrm{D}$. degree in Electronic Engineering from Queens University Belfast, U.K., in 2004. He is currently a member of academic staff in the Institute of Electronics, Communications and Information Technology (ECIT) at the same university and an Associate Researcher at LInstituit National des Sciences Appliques (INSA) Rennes, France. His research interests are in the design and implementation of embedded data, signal and image processing applications. He is Vice-Chair of the IEEE Technical Committee on Design and Implementation of Signal Processing Systems (DISPS), Area Editor of IEEE Transactions on Signal Processing and was founding Chief Technology Officer (CTO) of Analytics Engines Ltd. He was founding Chief Editor of the IEEE Signal Processing Society Resource Center and is a member of the Editorial Board of Springers Journal of Signal Processing Systems. 